Oh. J. et. al.

iTEC-based thymic rejuvenation

\title{
Thymic rejuvenation via induced thymic epithelial cells (iTECs) from FOXN1-overexpressing fibroblasts to counteract inflammaging
}

\author{
Jiyoung Oh, Weikan Wang, Rachel Thomas, and Dong-Ming Su*
}

\begin{abstract}
Department of Microbiology, Immunology, \& Genetics, University of North Texas Health Science Center, Fort Worth, TX, 76107, USA.
\end{abstract}

Running Title: iTEC-based thymic rejuvenation counteracts inflammaging

Key words: Aging, Thymic involution; Inducible FoxN1-overexpression; Aging-associated chronic inflammation; Rejuvenation

\section{* Corresponding Author: Dong-Ming Su}

Department of Microbiology, Immunology, \& Genetics, University of North Texas Health Center, 3500 Camp Bowie Blvd. Fort Worth, TX, 76107, USA.

Tel. 1-817-735-5186, Fax: 1-817-735-2118,

E-mail address: dongming.su@unthsc.edu

Abbreviations: Aire: autoimmune regulator gene; BM: bone marrow; CD4 ${ }^{\text {SP }}$ : CD4 ${ }^{+}$CD8 ${ }^{\text {-neg }}$ single positive; CreER $^{\text {T: }}$ Cre-recombinase and estrogen-receptor fusion protein; cTEC/mTEC: cortical/medullary thymic epithelial cells; FTg: STOP flox $-F o x N 1$ cDNA transgene; GFP: green fluorescent protein; iTECs: induced TECs from inducible FOXN1-overexpressing embryonic fibroblasts; MEF: mouse embryonic fibroblast; mOVA the membrane-bound chicken ovalbumin driven by the rat insulin promoter (RIP); OT-II CD4 ${ }^{+}$T cells: MHC class-II restricted and OVA recognizing T cell receptor transgenic $\mathrm{CD}^{+}{ }^{+} \mathrm{T}$ cells; R26: Rosa26 gene; Rag: $V(D) J$-recombination-activating gene; SASP: senescence-associated secretory phenotype; SP: single positive; TCR: T cell receptor; Tg: transgenic; TM: tamoxifen; WT: wild type.

Funding: This work was supported by NIH/NIAID grant R01AI121147 to D-M. S. 
Oh. J. et. al.

iTEC-based thymic rejuvenation

\section{Abstract}

Age-associated systemic, chronic, sterile inflammatory condition (inflammaging) is partially

34 attributed to increased self (auto)-reactivity, resulting from disruption of central tolerance in the aged,

35 involuted thymus. Age-related thymic involution causally results from gradually declined expression of the

36 transcription factor forkhead box N1 (FOXN1) in thymic epithelial cells (TECs), while exogenous FOXN1

37 in TECs can significantly rescue age-related thymic involution. Given the findings that induced TECs

38 (iTECs) from FOXN1-overexpressing embryonic fibroblasts can generate an ectopic de novo thymus under

39 the kidney capsule and intra-thymically injected natural young TECs can lead to middle-aged thymus

40 regrowth, we sought to expand upon these two findings by applying them as a novel thymic rejuvenation

41 strategy with two types of promoter-driven (Rosa26CreER ${ }^{\mathrm{T}}$ and FoxN1Cre) Cre-mediated iTECs. We

42 engrafted iTECs, rather than natural young TECs, directly into the aged thymus and/or peri-thymus and found

43 a significantly rejuvenated architecture and function in the native aged murine thymus. The engrafted iTECs

44 drove regrowth of the aged thymus in both male and female mice, showing not only increased thymopoiesis,

45 but also reinforcement of thymocyte negative selection, thereby, reducing senescent $\mathrm{T}$ cells and auto-reactive

46 T cell-mediated inflammaging phenotypes in old mice. Therefore, this is a promising thymic rejuvenation

47 strategy with preclinical significance, which can potentially rescue declined thymopoiesis and impaired

48 negative selection to significantly, albeit partially, restore the defective central tolerance and reduce

49 subclinical chronic inflammatory symptoms in the elderly. 
FTg mouse

Rosa26

locus:

Ex1

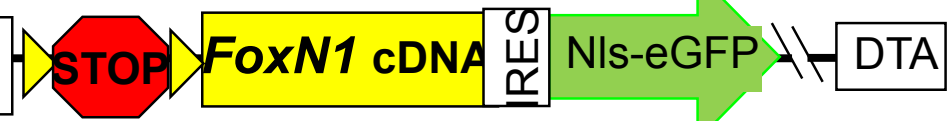

crossbreeding

Rosa26-CreER ${ }^{\top}$ mouse

OR

FoxN1-Cre mouse

$\downarrow$ Isolated from embryonic $\downarrow$

$13-14$ days

$+4-\mathrm{OHT}(\mathrm{TM})$

(cleaving

floxed-STOP)

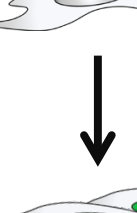

MEFs

iTEC line-2

(FTg-only)

(Control-line)

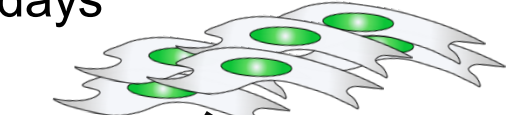

(FoxN1-GFP)
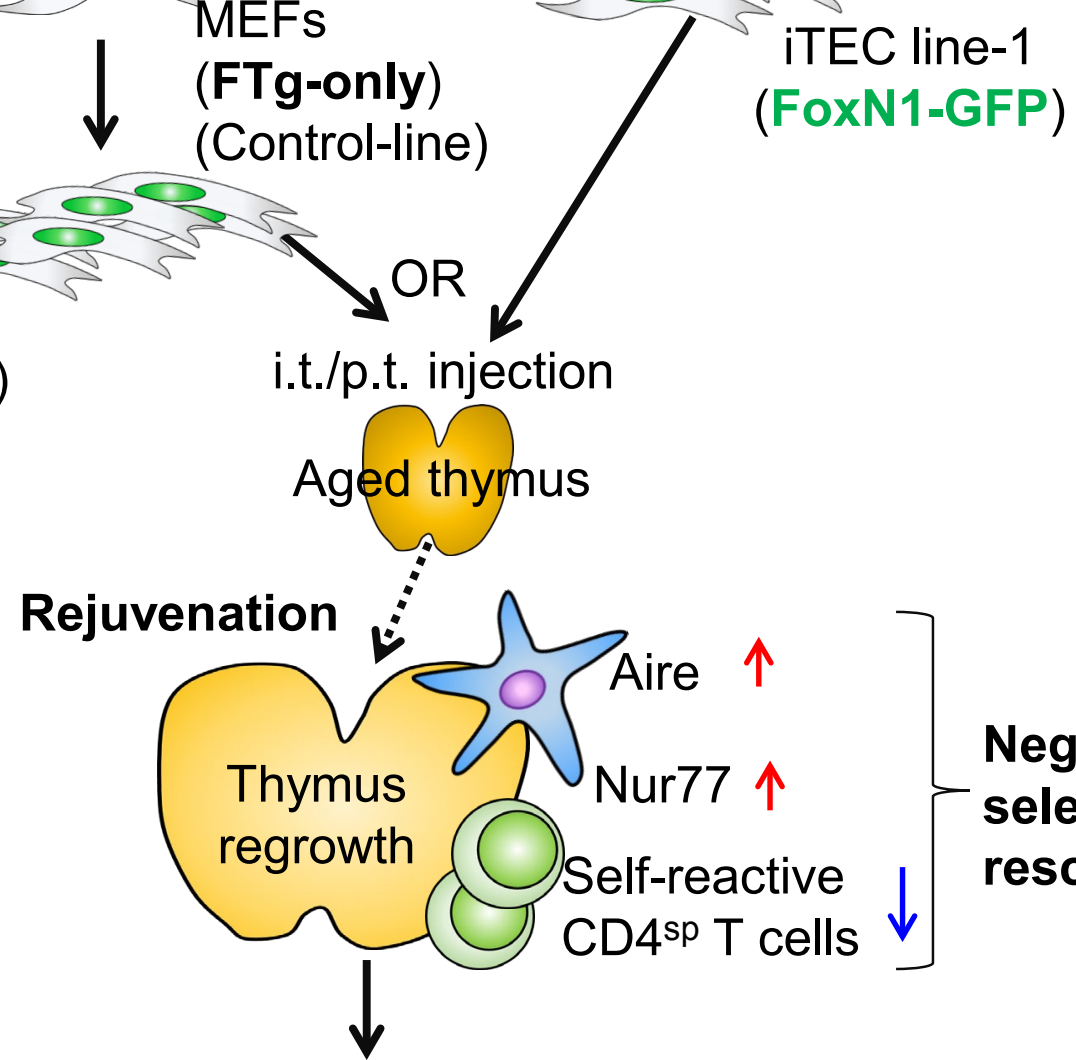

Negative

selection

rescued

PD-1/ CD153 expression (Immunosenescence) $\downarrow$

IL-6, IL-1 $\beta$, Lymphocyte infiltration (Inflammaging) $\downarrow$

Graphical Abstract: A novel rejuvenation strategy via the FOXN1-TEC axis using induced two types of FOXN1overexpressing embryonic fibroblasts (termed iTECs) by intrathymic injection is able to counteract age-related thymic involution, which rescued negative selection, thereby, reducing peripheral $\mathrm{T}$ cell-associated inflammaging conditions. 


\section{INTRODUCTION}

Age-related immune dysfunction is generally characterized by two extremes: immunosenescence (immune insufficiency) (McElhaney \& Effros 2009) and inflammaging (a chronic, persistent, sterile systemic inflammation, partially due to strong self-reactivity) (Freund et al. 2010; Franceschi \& Campisi 2014). These are antagonistic phenotypes, but they actually comprise two sides of the same coin (Fulop et al. 2017), and are associated with functional defects in the aged, atrophied thymus (Goronzy \& Weyand 2012; Xia et al. 2012; Coder et al. 2015; Palmer et al. 2018). Immunosenescence, unlike cultured cellular senescence, happens at systemic levels exhibiting diminished immune reaction in response to antigen stimulations, mainly due to contracted T cell receptor (TCR) repertoire diversity (Vallejo 2006). This is primarily attributed to a declined output of naïve T cells from the aged, atrophied thymus (Hale et al. 2006) and expansion of monoclonal memory $\mathrm{T}$ cells in the periphery (Detailed in our review) (Thomas et al. 2020). Although inflammaging was originally attributed to somatic cell senescence-associated secretory phenotype (SASP) (Coppe et al. 2010) and chronic innate immune activation (Fulop et al. 2017; Fulop et al. 2018), the contribution of aged adaptive immune components and specifically self-reactive $\mathrm{T}$ lymphocytes, as a probable primary contributor, has been recently determined (Coder et al. 2015; Fulop et al. 2018). The increased self-reactive $\mathrm{T}$ cells in the elderly are derived from perturbed central $\mathrm{T}$ cell tolerance establishment (Xia et al. 2012; Coder et al. 2015; Klein et al. 2019), due to defects in negative selection and altered regulatory T (Treg) cell generation (Coder et al. 2015; Oh et al. 2017) in the aged, atrophied thymus.

During aging the thymus undergoes a progressive, age-related atrophy, or involution, and a key diminished expression of transcription factor forkhead box N1 (FOXN1) in TECs (Ortman et al. 2002; Sun et al. 2010; Rode et al. 2015). Therefore, in order to ameliorate immunosenescence and reduce inflammaging through restoration of the aged $\mathrm{T}$ cell immune system, many have focused on targeting TECs in the aged thymus. Since the TEC-autonomous factor FOXN1 is heavily implicated in onset and progression of agerelated thymic involution, currently more strategies for rejuvenation of the aged thymic concentrate on the FOXN1-TEC axis, although there are strategies other than FOXN1-based, such as growth/sex hormones (Detailed in our review) (Thomas et al. 2020). FOXN1-TEC axis strategies includes $F$ oxN $1^{\mathrm{eGFP} / \mathrm{+}}$ knockin epithelial cells (Barsanti et al. 2017), newborn TEC-based intrathymic injection (Kim et al. 2015), inducible FoxN1-expressed mouse embryonic fibroblast (MEF)-based ectopic thymus generation (Bredenkamp et al. 2014b), and genetically-based rejuvenation via enhancement of exogenous FoxN1 expression with FoxN1 cDNA plasmid (Sun et al. 2010) and FoxN1 transgene (Tg) in TECs (Zook et al. 2011; Bredenkamp et al. 2014a). In addition, cytokine/growth factor-to-TEC based rejuvenation strategies have been studied, including addition of mesenchymal cell-derived keratinocyte growth factor (KGF) (Min et al. 2007), macrophage- and T lymphocyte-derived insulin-like growth factor-1 (IGF-1) (Chu et al. 2008), thymic stromal cell-derived bone morphogenetic protein-4 (BMP4) (Tsai et al. 2003; Wertheimer et al. 2018), and lymphoid tissue inducer (LTi) cell-derived IL-22 (Dudakov et al. 2012). These factors are produced from cells of mesenchymal or hematopoietic origin, but target non-hematopoietic TECs associated with up-regulating FoxN1 expression in TECs. Finally, epigenetically-based rejuvenation, via extracellular vesicles and exosomes extracted from young healthy serum has been shown to rejuvenate not only the peripheral T cell system, but also the thymus by enhancing FoxN1 expression (Wang et al. 2018). Therefore, there is potential for rejuvenating thymic aging by primarily targeting the restoration of TEC homeostasis through rescuing age-related declined FoxN1 expression.

Among the FOXN1-TEC axis therapies for thymic rejuvenation, two strategies are particularly attractive. One strategy is to aggregate induced Rosa26(R26)CreER ${ }^{\mathrm{T}}$-mediated $F O X N 1$-overexpressed MEFs (converting these cells into pseudo-TECs, termed induced TECs or iTECs) along with early-stage thymocytes and fetal mesenchymal cells to build an ectopic thymus under the kidney capsule of adult mice (Bredenkamp et al. 2014b). This de novo ectopic thymus produced functional T cells. However, one limitation is that the aged, native thymus remains in the host releasing self-reactive $\mathrm{T}$ cells that still contribute to inflammaging. The other strategy is an intrathymic injection of freshly isolated newborn TECs (non-manipulated TECs), in which FoxN1 is normally highly expressed, into the native thymus of middle-aged mice (Kim et al. 2015). This led to restoration of thymopoiesis. However, collection of fresh newborn TECs is not feasible when considering translating this rejuvenation strategy to humans, and isolation of fresh TECs without thymocyte 103 contamination is very difficult since TECs and their progenitors comprise a miniscule portion of the thymus 
(Ulyanchenko et al. 2016). Therefore, these promising thymic rejuvenation strategies for development of a practical therapy contain several limitations.

Fortunately, fibroblasts, which could be very easily isolated from human patients, can be engineered to overexpress FOXN1 for induction of iTECs for intra-/peri-thymic injection. Based on these scientific premises, we expanded on these two findings and applied them to develop a novel thymic rejuvenation strategy. We directly engrafted iTECs into the aged, native thymus to rejuvenate function of the aged, native thymus and assessed this in a mouse model, by using MEFs from our engineered STOPflox-FoxN1 transgenic (FTg) mouse allele (Zhang et al. 2012; Ruan et al. 2014) (Supplemental Fig. S1), mediated by two types of promoter-driven (Rosa26CreER ${ }^{\mathrm{T}}$ and FoxNlCre) Cre-recombinase.

We found that the engrafted iTECs drove regrowth of the aged thymuses in both male and female mice with increased thymopoiesis and improved thymic architecture. These led to a reinforcement of thymocyte negative selection in the native, aged thymus, thereby attenuating auto-reactive $\mathrm{T}$ cell-mediated inflammaging phenotypes and reducing senescent $\mathrm{T}$ cells in old mice. Although the native, aged thymus cannot fully return to young levels in our system, this is a promising thymic rejuvenation strategy with preclinical significance to counteract inflammaging.

\section{RESULTS}

\section{Preparation and characterization of iTECs}

A previous report demonstrated that enforced FOXN1 expression in MEFs from embryos generated by crossbreeding of STOP flox $-F o x N 1$ transgenic and R26-CreER ${ }^{\mathrm{T}}$ mice induced epithelial characteristics in fibroblasts (Bredenkamp et al. 2014b). Since we generated similar STOPflox $-F o x N 1$ transgenic (exogenous FoxN1 cDNA driven by R26 promoter, termed FTg) mice (DNA construct is shown in Supplemental Fig. S1) (Zhang et al. 2012; Ruan et al. 2014), we crossbred these mice with either R26-CreER ${ }^{\mathrm{T}}$ or FoxN1-Cre mice to generate FTg:R26CreER $R^{\mathrm{T}}$ and FTg:FoxN1Cre embryonic mice, respectively. We confirmed epithelial characteristics in MEFs from two different promoter-driven FoxN1 expressing lines in our mouse colonies (Fig. 1). Using NIs-eGFP (nuclear localization signal enhanced green fluorescent protein) as an indicator of exogenous FoxN1 expression (Zhang et al. 2012; Ruan et al. 2014) in the cultured MEFs (isolated from embryonic day-13 (E13) and E14 mice), we found MEFs from FTg-only (without any Cre-Tg) and $\mathrm{FTg}: R 26 \mathrm{CreER}{ }^{\mathrm{T}}$ without addition of tamoxifen (xTM, 4-OHT) did not express GFP (Fig. 1A left panels) due to lack of activated Cre, while FTg:FoxN1Cre (TM not required) and FTg:R26CreER ${ }^{\mathrm{T}}$ lines treated with TM for 48 hours showed GFP expression (Fig. 1A right panels and Fig. 1B middle and right panels). We also found that MEFs with greatly increased exogenous FoxN1 expression from FTg:FoxN1Cre and FTg:R26CreER ${ }^{\mathrm{T}}$ (xTM) mice showed TEC identifying markers $\left(\mathrm{EpCAM}^{+}\right.$and MHC-II ${ }^{+}$cells in the $\mathrm{GFP}^{+}$ population) (Fig. 1B, middle and right panels), but not MEFs of FTg:R26CreER ${ }^{\mathrm{T}}$ without addition of TM (Fig. 1B, left panels).

Exogenous FoxN1 mRNAs were indeed increased in the two Cre-activated groups (Fig. 1C, two middle bars in leftmost panel). In addition, some TEC functional molecules, which are key effectors in promoting thymocyte development, such as Notch ligand Dll4 and thymus-expressed chemokine ligand $\mathrm{Ccl} 25$, were increased in MEFs with activated $\mathrm{Cre}$-Tg (Fig. 1C, middle and right panels). Notably, expression of both exogenous FoxN1 and effector molecules were increased in the iTECs, but their increased levels in these pseudo-TECs were still lower or similar to their expression in the natural newborn thymus, during which these molecules should be normally highly expressed (rightmost striped bars in Fig. 1C all panels). In addition, FoxN1Cre-mediated expression of exogenous FoxNl and effector molecules in the FTg:FoxN1Cre line was higher than $R 26 \mathrm{CreER}^{\mathrm{T}}$-mediated ones. This is probably due to $\mathrm{Cre}$-Tg turning on via endogenous FoxN1 in vivo, which is activated by E11.25 in the thymus (Gordon et al. 2001) and potentially in the E12.5 skin (Gordon et al. 2007) or alternatively at low levels in the E13.5 skin (Bredenkamp et al. 2014b) during the organogenesis of B6 mice. This in vivo endogenous FoxN1-induced exogenous FoxN1 expression is 48hrs earlier than in vitro TM-induced expression in the $\mathrm{FTg}: R 26 C r e E R^{\mathrm{T}}$ line. Together, Cre-induced expression of exogenous FoxN1 and TEC functional molecules in MEFs conferred TEC characteristics to these MEFs. Therefore, these MEFs were termed as iTECs. 


\section{Intra-/peri-thymic (i.t./p.t.) transplantation of iTECs drove aged thymus regrowth}

Previous reports demonstrated that intrathymic (i.t.) injection of fetal thymic cells, containing young TECs with high-levels of FoxN1 expression, into middle-aged (9-12 months old) mice drove recipient thymus growth and increased $\mathrm{T}$ cell production (Kim et al. 2015). Since Kim et al.'s approach requires a newborn thymus for rescuing an aged thymus and newborn TECs are difficult to obtain and purify, we tested whether our iTECs could yield similar outcomes in fully aged (over 18 months old) mice. We firstly examined thymus regrowth and thymopoiesis of aged mice ( $\sim 18$ months old at the time of the injection and 19 - 20 months old at the time of analysis) after i.t./p.t. transplantation of iTECs. Our results show that transplantation of iTECs indeed drove aged thymus regrowth (Fig. 2) exhibited by increased thymic size, weight, and thymocyte numbers (Figs. 2A, 2B, and 2C, respectively). These changes were the same in male and female mice. Although these improvements did not reach the same levels as the young mice (Fig. 2A, top row and Figs. $2 \mathrm{~B}$ and $\mathrm{C}$, leftmost group in each panel), it was significantly improved compared to the naturally aged group without transplantation of iTECs (transplantation of FTg-only MEFs served as a negative control allowing for the same surgical stress as the iTEC-engrafted groups).

Overall, our iTECs better resemble newborn TECs and more efficiently drive the aged ( $\geq 18$ months old), atrophied thymus regrowth and rejuvenation of thymopoiesis. It appears that the efficacy from both iTEC lines were generally similar, but endogenous FoxN1 promoter-driven Cre was slightly better than $R 26$ promoter-driven CreER ${ }^{\mathrm{T}}$ (xTM) (See Fig. $2 \mathrm{C}$ rightmost two groups in the rightmost panel). This could be explained by the fact that although $R 26 \mathrm{CreER}^{\mathrm{T}}$ is turned on in vitro during the culture with TM-induction, which is 48 hours later than the FoxN1Cre is activated in vivo. Expression of the effector molecules in FTg:R26CreER ${ }^{\mathrm{T}}$ line was lower than FTg:FoxN1Cre line (Fig. 1C), but they could become the equivalent after injection into the host thymuses, since the effector molecules probably increase only to a homeostatic plateau.

\section{Grafted iTECs rejuvenated thymic architecture in aged mice}

Increased thymic mass (Figs. 2A and B) generally reflects expansion in thymocytes (Fig. 2C) and regrowth in TECs, because rejuvenation of TEC meshwork is essential for thymocyte regrowth. We examined TEC-based thymic microstructure using TEC-associated markers (Fig. 3). After co-staining with keratin-5 (K5, red) (medullary region) and K8, (cortical region) (all in green in Fig. 3), the aged, atrophied thymus showed disorganized and reduced $\mathrm{K}^{+}$regions (Fig. 3A, the second panel from left). After treatment with either FTg:FoxN1Cre or FTg:R26CreER ${ }^{\mathrm{T}}$ (xTM) iTECs (Fig. 3A, right 2 panels), the $\mathrm{K}^{+}$regions became organized, similar to the young thymus (Fig. 3A, leftmost panel). Increased UEA-1 ${ }^{+}$TECs showed the well-organized medulla, exhibiting the same trends as the $\mathrm{K}^{+}$region (Fig. 3B). Claudin (Cld)-3 and -4 $(\mathrm{Cld} 3+4)$ are immature medullary thymic epithelial cells (mTEC) markers (Hamazaki et al. 2007; Sekai et al. 2014) and $\beta 5$ t is mainly expressed in immature cortical thymic epithelial cells (cTECs) (Ripen et al. 2011). These were decreased in the naturally aged thymus, but were rescued in the naturally aged thymus treated with either of the two promoter-driven Cre-induced iTECs (Figs. 3C and D). These results infer that input of iTECs enhances native TEC regrowth to rejuvenate aged thymic architecture, thereby improving thymic microenvironment and rebooting thymopoiesis.

To confirm whether the observed TECs regrew from the native aged thymus when they received stimulation from iTEC-rejuvenated microenvironment, or if these TECs grew directly from newly transplanted iTECs, we examined the sources of these TECs in the rejuvenated, aged thymuses based on endogenous and exogenous FoxN1 expression. The TECs with positive staining for FoxN1 using rabbit antiFoxN1 (the antibody was kindly provided by Dr. Itoi, Japan) (Itoi et al. 2007) exhibited only endogenous FoxN1, while the TECs with both antibody-specific FoxN1 staining and FTg-GFP (See supplemental Fig.S1) expression (double positive) contain exogenous FoxN1 and would therefore be derived from the newly transplanted iTECs. We found that both native TECs and transplanted iTECs were expanding within 10 days after the engraftment (Fig. 3E, right two-ranked panels on top and middle two rows), particularly in mTECs $\left(\mathrm{CD} 45^{- \text {-neg }}{ }^{\text {MHC-II }}{ }^{+}\right.$population, top row). Further, the transplanted iTECs exhibited reduced expansion but 
the native TECs were still robustly expanding over 20 days after the engraftment (Fig. 3E bottom row). The results suggest that although engrafted iTECs growth is transient, they do exhibit growth, and they can also promote native TEC growth in the recipient thymus even after their growth begins to wane. Thus, it seems that once native TECs receive necessary stimulation, they undergo a more prolonged expansion compared to the engrafted iTECs. However, both the engrafted iTECs and rejuvenated native TECs cooperate to restore the aged thymic microenvironment to promote thymocyte expansion.

\section{Engrafted iTECs expanded Aire-expressing mTECs, increased negative selection signaling in CD4 ${ }^{\mathrm{SP}}$ thymocytes, and restored declined thymocyte negative selection in the aged thymus}

Autoimmune regulatory, Aire, gene is expressed by mTECs to mediate self-antigen expression and promote central immune tolerance via thymocyte negative selection and Treg generation (Anderson et al. 2005; Anderson \& Su 2016). In the aged thymus Aire-expressing mTECs are disrupted and/or declined (Coder et al. 2015; Wang et al. 2018). Since transplantation of iTECs enhanced biological characteristics of native TECs in the naturally aged thymus (Fig. 3), we tested whether transplantation of the two iTEC lines was able to expand declined Aire-expressing mTECs and found positive results (Fig. 4A. bottom row) with statistical significance (Fig. 4B. two right groups) in the aged thymus.

Self (auto)-reactive thymocytes undergo negative selection dependent on TCR signaling strength, while the intensity of Nur77 expression in thymocytes reflects a negative selection signaling strength. We examined mean fluorescence intensity (MFI) of Nur77 in CD4 single positive (CD4 ${ }^{\mathrm{SP}}$ ) thymocytes from various groups (Fig. 4C), and found MFIs of Nur77 in CD4 $4^{\mathrm{SP}}$ thymocytes were indeed increased in the two iTEC-grafted groups (Fig. 4C, right two square-symbol groups in the right panel). Although these increases did not reach the same levels as in young mice (Fig. 4C, a filled-circle group in the right panel), they were significantly increased, compared to naturally-aged controls (FTg-only group).

The results provided an indication that transplantation of iTECs potentially restores TEC function in negative selection as demonstrated by increased Aire ${ }^{+}$mTECs and enhanced negative selection signaling strength in the $\mathrm{CD} 4^{\mathrm{SP}}$ thymocytes in the aged thymus. In order to obtain direct evidence that the declined thymocyte negative selection in the aged thymus is really restored, we designed an observable negative selection model, in which mOVA-Tg host young and aged mice were reconstituted with donor OT-II TCR$\mathrm{Tg}$ mouse bone marrow (BM) cells. This is a well-designed thymocyte negative selection model, in which a neo-self-antigen mOVA presented on mTECs induces OT-II TCR-Tg CD4 $4^{\text {SP }}$ thymocyte depletion (negatively selected), able to be observed through flow cytometry assay (Hubert et al. 2011; Coder et al. 2015). The thymuses in the immune system-reconstituted young and aged mice were engrafted with FTg:FoxN1Cre iTECs or control FTg-only MEFs. Four weeks after the transplantation of these cells, the proportion of OT-II-specific CD4 ${ }^{\mathrm{SP}}$ thymocytes was determined (Fig. 5A). Increased proportion of OT-IIspecific $\mathrm{CD} 4{ }^{\mathrm{SP}}$ thymocytes in the mOVA-Tg thymic microenvironment means defective negative selection, which was seen in the aged, atrophied thymus (Fig. 5B middle panels, and Fig. 5C middle bar). However, this proportion was reduced after transplantation with iTECs (Fig. 5B right panels and Fig. 5C rightmost bar) in the aged mOVA-Tg thymuses. Meanwhile, signaling of negative selection (Nur77) in the specific CD4 ${ }^{\mathrm{SP}}$ thymocytes was increased (Figs. 5D yellow histogram and 5E rightmost bar). The results imply that engrafted iTECs were indeed able to significantly restore mTEC-mediated function for self-reactive thymocyte negative selection in the aged, atrophied thymus.

\section{Engrafted iTECs counteracted inflammaging by exhibiting reduced inflammatory cytokines and lymphocyte infiltration into non-lymphoid organs in the periphery}

To confirm whether the restoration of negative selection in the iTEC-engrafted aged thymus could counteract inflammaging-associated phenotypes in the aged periphery, we examined the levels of inflammatory cytokines and lymphocyte infiltration into non-lymphoid organs through adoptive transfer of rejuvenated spleen cells from rejuvenated mice. As we know, inflammaging is attributed to not only senescence somatic cells producing SASP and chronic innate immune cell activation, but also self (auto)- 
reactive $\mathrm{T}$ cell-induced self-tissue damages. These self-reactive $\mathrm{T}$ cells are released from the aged, atrophied thymus due to defective negative selection (Goronzy \& Weyand 2012; Xia et al. 2012; Coder et al. 2015; Palmer et al. 2018). If the engrafted iTECs can restore declined negative selection, the self-reactive $\mathrm{T}$ cells released should be reduced, and thereby, peripheral inflammaging-associated phenotypes should be attenuated.

\section{Engrafted iTECs indirectly reduced senescent $T$ cells and enhanced $T$ cell immune response in the periphery of aged mice}

Inflammaging is also partially attributed to immunosenescence because senescent/ exhausted peripheral $\mathrm{T}$ cells not only produce inflammatory factors but are also unable to properly clear senescent somatic cells, which produce SASP (Prata et al. 2019; Thomas et al. 2020). We asked whether iTEC-driven rejuvenation of aged thymic function could counteract inflammaging through reducing senescent $\mathrm{T}$ cells associated with increased output of newly-generated $\mathrm{T}$ cells, since the rejuvenated thymus increases thymopoiesis (Fig. 2). We found that 45 days after iTEC engraftment senescent CD4 ${ }^{\mathrm{SP}} \mathrm{T}$ cells $\left(\mathrm{CD} 4^{+} \mathrm{PD}-\right.$ $1^{+} \mathrm{CD} 153^{+}$) (Shimatani et al. 2009; Tahir et al. 2015) were significantly reduced in the periphery of aged mice (Supportive Figs. S2A and B, right two panels and right two bars), compared to the aged mice which received FTg-only MEFs.

In addition, we also verified the peripheral $\mathrm{CD} 4{ }^{\mathrm{SP}} \mathrm{T}$ cell response to co-stimulation from $\mathrm{CD} 3 \varepsilon$ and CD28 antibodies. This response, represented by intracellular IL-2 mean fluorescence intensity (MFI) (Fig. $\mathrm{S} 2 \mathrm{C}$ ), was declined in peripheral CD4 ${ }^{\mathrm{SP}} \mathrm{T}$ cells of aged individuals (Fig. S2D, the $2^{\text {nd }}$ bar from the left) (Sun et al. 2010), but was significantly restored in peripheral CD4 ${ }^{\mathrm{SP}} \mathrm{T}$ cells from iTEC-rejuvenated mice (Fig. S2D, two bars with filled and opened square symbols), implying increased proportion of newly-generated $\mathrm{T}$ cells in the rejuvenated mice. Taken together, iTEC-driven changes in the aged thymus could additionally confer a positive rejuvenation effect on the peripheral $\mathrm{T}$ cell system.

\section{DISCUSSION}

$\mathrm{T}$ cell-mediated adaptive immunity during aging is intricately involved in both immunosenescence and inflammaging. One of the potential strategies for ameliorating these two extremes is rejuvenation of the aged, involuted thymus. Restoring thymic function of central tolerance establishment via repairing the defects in negative selection is critical for counteracting inflammaging. Although there are many strategies for rejuvenation of 
thymic involution, targeting defective TEC homeostasis via the FOXN1-TEC axis is one of the most effective strategies.

We tested an application of cellular rejuvenation of age-related thymic involution

The underlying mechanism of thymic rejuvenation potentially involves restoration
$C$ regrowth in the aged thymus via both expanded engrafted iTECs (increased by using iTECs generated from $R 26 \mathrm{CreER}^{\mathrm{T}}$ and FoxN1Cre-induced exogenous FoxN1 in $S T O P^{\text {flox }}$ FoxN1-Tg embryonic fibroblasts with intrathymic injection. We found that the engrafted iTECs were able to induce aged thymus regrowth with increased thymopoiesis in aged male and female mice (Fig. 2), in which native TECs were reorganized (Figs. 3A$\mathrm{D}$, right two columns) and underwent expansion (Fig. 3E, right two columns). We also observed reinforced thymocyte negative selection (Figs. 4 and 5). This resulted in reduced auto-reactive $\mathrm{T}$ cell-mediated inflammaging-associated phenotypes and diminished peripheral senescent $\mathrm{T}$ cells in the aged periphery (Figs. 6 and S2). of TEC regrowth in the aged thymus via both expanded engrafted iTECs (increased
GFP $^{+} \mathrm{FoxN} 1^{+}$, double positive, TECs) and induced expansion of native TECs (increased $\mathrm{GFP}^{- \text {neg }} \mathrm{FoxN1}^{+}$, single positive, TECs) (Fig. 3E, right two columns). This improves the aged thymic microenvironment, promoting normal thymocyte homeostasis and development. These effects culminated in attenuation of inflammaging phenotypes (Fig. 6) and removal of senescent T cells (Figs. S2A and S2B). Although we did not directly measure native $\mathrm{T}$ cells ( $\mathrm{T}$ cells generated prior to iTEC engraftment), we found that $\mathrm{T}$ cells from rejuvenated mice exhibited an increased response to TCR stimulation (Figs. S2C and $\mathrm{S} 2 \mathrm{D})$, which is a functional sign of healthy newly-generated T cells.

Although the rejuvenation was partial, since it cannot restore to the same levels as in young mice, it was significant when compared to the same aged counterparts treated with non-exogenous FoxN1-expressing MEFs. The effects of a one-time transplantation of these cells is also most likely transient, since the engrafted iTECs are not TEC stem cells and therefore do not demonstrate unlimited growth after engraftment in the aged, native thymus. Compared to the generation of an ectopic de novo thymus with induced FOXN1overexpressing MEFs under the kidney capsule of adult mice (Bredenkamp et al. 2014b) and intrathymic injection of newborn TECs into the middle-aged thymus (Kim et al. 2015), our strategy facilitates a more clinically translational rejuvenation therapy. Although an ectopic de novo thymus can generate naïve T cells, this does not remedy the increased selfreactive $\mathrm{T}$ cells released by the native atrophied thymus remaining in the aged host. In addition, intrathymic injection of newborn TECs can rejuvenate middle-aged thymus in mice (Kim et al. 2015), but the source of newborn TECs for human treatment is limited. Further, our rejuvenation effects were observed in aged mice ( $\geq 18$ months old) rather than limited to middle-aged mice (Kim et al. 2015).

In comparison with exogenous FoxN1 expression and rejuvenation effects from two promoter-driven Cre-Tg (FoxN1Cre and R26CreER ${ }^{\mathrm{T}}$ )-mediated iTECs, exogenous FoxN1 expression was slightly higher in the former cell type (Fig. 1C leftmost panel), and the effects were not that different between the two lines (Figs. 2-4). We think that this is probably due to the length of time for which the exogenous FoxN1-Tg has been activated. It has been turned on in vivo before their isolation, because endogenous FoxN1-driven-Cre could have been already activated in the E13 and E14 MEF cells, whereas, the exogenous FoxN1-Tg expression mediated by $R 26 \mathrm{CreER}^{\mathrm{T}}$ is turned on after dissection and during the 
48-hour culture with TM induction, i.e. 48 hours later than the former. However, the effector molecules (Dll4 and Ccl25) most likely reach a homeostatic plateau. Once these two lines are injected into host mice, expression of the effector molecules in the $R 26 \mathrm{CreER}^{\mathrm{T}}$ line could feasibly "catch up" to the levels expressed by the former line. In addition, FoxN1Cre mediates exogenous FoxN1 expression only in skin epithelial cells of MEFs, while $R 26 C r e E R^{\mathrm{T}}$ mediates exogenous FoxN1 expression in most tissues, including fibroblasts and epithelial cells of MEFs, since the $R 26$ promoter is ubiquitous. Thus, it is not surprising that the effects from both lines are similar. The results imply that this cellular therapeutic strategy is highly clinically translational, since fibroblasts derived directly from patients themselves, who would be treatment recipients, can be readily targeted for genetic engineering of FoxNl expression.

In sum, our preliminary, proof-of-principle, cellular-based rejuvenation strategy via the FOXN1TEC axis with intra-/peri-thymic injection is a promising thymic rejuvenation strategy with potential clinical significance. Once the application study is further formulated and investigated, intrathymic transplantation of genetically engineered FoxN1-expressing patient skin cells (fibroblasts) could facilitate attenuation of T cell immunosenescence and subclinical chronic inflammatory symptoms in the elderly.

\section{Experimental Procedures}

\section{Animal models}

C57BL/6 genetic background mouse models were used. Wild-type (WT) young and aged mice were

\section{Preparation of MEFs for intrathymic injection} from our breeding colonies and National Institute on Aging (NIA) aged rodent colonies. STOP ${ }^{\text {flox }}$-FoxN1 transgenic (FTg) mice were generated in our lab previously (Zhang et al. 2012; Ruan et al. 2014) (Supportive Figure S1) and were crossbred with either R26-CreER ${ }^{\mathrm{T}}$ mice (Jackson Lab \#004847) or FoxN1-Cre mice (Jackson Lab \#018448) for the generation of FTg:R26CreER ${ }^{\mathrm{T}}$ [tamoxifen (TM)-inducible exogenous FoxN1 overexpression in the R26-expressing tissues] and FTg:FoxN1Cre [exogenous FoxN1 overexpression induced by endogenous FoxN1 promoter-driven Cre-Tg (Gordon et al. 2007)] embryonic mice, respectively. Other genetically engineered mouse colonies were RIP-mOVA [the rat insulin promoter (RIP)-driven membrane-bound ovalbumin] Tg mice (Jackson Lab \#005431); OT-II ${ }^{+}$TCR-Tg (transgenic TCR recognizing ovalbumin in the context of MHC-class II, I-A ${ }^{\mathrm{b}}$ ) mice (Jackson Lab \#004194); and Rag $^{-/}$(Ragl gene knockout) mice (Jackson Lab \#002216). Mouse ages are indicated in each figure legend, or defined as young (1 - 2 months old) and naturally-aged ( \pm 18 months old). All animal experiments were performed in compliance with protocols approved by the Institutional Animal Care and Use Committee of the University of North Texas Health Science Center, following guidelines of the National Institutes of Health.

MEFs were prepared from E13 and 14 embryonic mice (the gestation day-0 "E0" was determined by the presence of a vaginal plug in the first morning on the mother mouse). All the organs of the embryonic mice were removed except for the body with skin, which was trypsinized with Trypsin-EDTA solution to generate single-cell suspensions. Cells were cultured in 10\%FBS/DMEM medium, with $2 \mathrm{mM}$ L-glutamine,

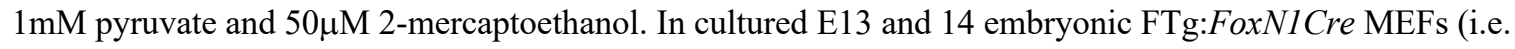
one type of iTEC donor cells), exogenous FoxNl is consistently expressed, due to endogenous FoxNl-driven Cre having been turned on, which was found at part of the skin of E12.5 embryonic mice (Gordon et al. 2007), and spontaneously activated at low levels, which was observed at E13.5 MEFs (Bredenkamp et al. 2014b). For inducing exogenous FoxN1 overexpression in FTg:R26CreER ${ }^{\mathrm{T}} \mathrm{MEFs}$ (i.e. another type of iTEC donor cells), $1 \mu \mathrm{M}$ of 4-hydroxy tamoxifen (TM) (4-OHT) was added in the culture for 48hr. Exogenous FoxN1 overexpression [based on green fluorescent protein (GFP) expression] in the two types of MEF lines 
was examined after $48 \mathrm{hr}$ culture. These two cell lines were expanded with two passages. We used the third passage cells as injection reagents. FTg-only (without any Cre Tg) MEFs (control donor cells) were used as negative control. All embryonic mice for preparation of MEFs were genotyped. All cells were checked for GFP expression prior to engraftment.

\section{Intra-/peri-thymic (i.t./p.t.) injection of donor cells into recipient mice}

FTg-only MEFs (negative control) and two types of promoter-driven Cre-mediated FTg iTECs were injected at $1 \times 10^{6}$ cells in $20 \mu$ l of volume per recipient mouse (young or naturally aged) into the thymus and/or peri-thymus in three locations via a suprasternal notch surgery under anesthesia (Burnley et al. 2013). Fortyfive days after the injection, the tissues of the recipient mice were analyzed for various phenotypes. More details about the operation are depicted in Supportive Experimental Procedures.

\section{Bone marrow (BM) adoptive transfer for assessing negative selection}

Erythrocyte-depleted and mature T cell-depleted (via anti-CD3 MACS beads and columns, Miltenyi Biotech) BM cells from OT-II ${ }^{+}$TCR-Tg mice, which carry a copy of CD45.1 congenic marker, were intravenously (i.v.) injected into recipient young or aged mOVA-Tg mice at $5 \times 10^{6}$ cells per recipient mouse, which had received irradiation at doses of $\sim 900 \mathrm{Rad}$. Two weeks after the BM cell transfer, FTg-only MEFs and FTg:FoxN1Cre iTECs were intrathymically (i.t.) injected into the thymus/peri-thymus of the recipient mOVA-Tg mice. Four weeks after the engraftment, the thymuses of the recipient mOVA-Tg mice were dissected for analysis of negative selection (proportion of $\mathrm{CD} 4{ }^{\mathrm{SP}}$ and MFI of Nur77 in $\mathrm{CD} 4^{\mathrm{SP}}$ ).

\section{Transplantation of splenocytes into Rag $^{-/}$recipients for assessing lymphocyte infiltration}

Protocol per our previous publication (Coder et al. 2015): briefly, erythrocyte-depleted splenocytes from FTg-only MEF- or FTg:FoxN1Cre iTEC-engrafted young or aged WT mice were i.v. injected at $2.5 \mathrm{x}$ $10^{7}$ cells per recipient mouse into the young recipient $\mathrm{Rag}^{-/-}$mice. Eight weeks after the transplantation, the salivary glands from the young recipient $\mathrm{Rag}^{-/-}$mice were analyzed for lymphocyte inflammatory infiltration with Hematoxylin and Eosin (H\&E) staining in paraffin sections ( $5 \mu \mathrm{m}$ thick).

\section{General analysis methods:}

Detailed analysis methods (Real-time RT-PCR, flow cytometer, immunofluorescence staining, and ELISA, etc.), as well as reagents are described in Supportive Experimental Procedures.

\section{Statistics}

Either the unpaired two-tailed Student's $t$-test for comparing two groups with equal variance or one-way ANOVA with Bonferroni correction for comparing multiple groups were employed. Differences were considered statistically significant at values of $* p<0.05$; $* * p<0.01 ; * * p<0.001$. All statistics were analyzed with Prism-8 software (GraphPad). 
Oh. J. et. al.

iTEC-based thymic rejuvenation

\section{Author contribution:}

426 J.O. designed and performed the most experiments, analyzed data, prepared figures, and wrote the 427 manuscript; W.W. performed most of the hands-on animal work; R.T. performed part of the experiments, 428 helped to write and proofread the manuscript; D-M.S. conceived, designed, and supervised the project, helped 429 with hands-on animal work, analyzed data, and wrote the manuscript.

\section{Author declaration:}

432 All authors have no conflicts of financial interests associated with this manuscript.

434 Funding: Supported by NIH/NIAID grant R01AI121147 to D-M. S. The funder had no role in study design, 435 data collection and analysis, decision to publish, or preparation of the manuscript. 
Oh. J. et. al.

iTEC-based thymic rejuvenation

\section{References}

Anderson MS, Su MA (2016). AIRE expands: new roles in immune tolerance and beyond. Nat Rev Immunol. 16, 247-258.

Anderson MS, Venanzi ES, Chen Z, Berzins SP, Benoist C, Mathis D (2005). The cellular mechanism of

Barsanti M, Lim JM, Hun ML, Lister N, Wong K, Hammett MV, Lepletier A, Boyd RL, Giudice A, Chidgey AP (2017). A novel Foxn1(eGFP/+) mouse model identifies Bmp4-induced maintenance of Foxn1 expression and thymic epithelial progenitor populations. Eur J Immunol. 47, 291-304.

Bredenkamp N, Nowell CS, Blackburn CC (2014a). Regeneration of the aged thymus by a single transcription factor. Development. 141, 1627-1637.

Bredenkamp N, Ulyanchenko S, O'Neill KE, Manley NR, Vaidya HJ, Blackburn CC (2014b). An organized and functional thymus generated from FOXN1-reprogrammed fibroblasts. Nat Cell Biol. 16, 902-908.

Burnley P, Rahman M, Wang H, Zhang Z, Sun X, Zhuge Q, Su DM (2013). Role of the p63-FoxN1 regulatory axis in thymic epithelial cell homeostasis during aging. Cell Death Dis. 4, e932.

Chu YW, Schmitz S, Choudhury B, Telford W, Kapoor V, Garfield S, Howe D, Gress RE (2008). Exogenous insulin-like growth factor 1 enhances thymopoiesis predominantly through thymic epithelial cell expansion. Blood. 112, 2836-2846.

Coder BD, Wang H, Ruan L, Su DM (2015). Thymic Involution Perturbs Negative Selection Leading to Autoreactive T Cells That Induce Chronic Inflammation. J Immunol. 194, 5825-5837.

Coppe JP, Desprez PY, Krtolica A, Campisi J (2010). The senescence-associated secretory phenotype: the dark side of tumor suppression. Annu Rev Pathol. 5, 99-118.

Dudakov JA, Hanash AM, Jenq RR, Young LF, Ghosh A, Singer NV, West ML, Smith OM, Holland AM, Tsai JJ, Boyd RL, van den Brink MR (2012). Interleukin-22 drives endogenous thymic regeneration in mice. Science. 336, 91-95.

Franceschi C, Campisi J (2014). Chronic inflammation (inflammaging) and its potential contribution to age-associated diseases. J Gerontol A Biol Sci Med Sci. 69 Suppl 1, S4-9.

Freund A, Orjalo AV, Desprez PY, Campisi J (2010). Inflammatory networks during cellular senescence: causes and consequences. Trends Mol Med.16, 238-246.

Fulop T, Larbi A, Dupuis G, Le Page A, Frost EH, Cohen AA, Witkowski JM, Franceschi C (2017). Immunosenescence and Inflamm-Aging As Two Sides of the Same Coin: Friends or Foes? Front Immunol. 8, 1960.

Fulop T, Witkowski JM, Olivieri F, Larbi A (2018). The integration of inflammaging in age-related diseases. Semin Immunol. 40, 17-35.

Gordon J, Bennett AR, Blackburn CC, Manley NR (2001). Gcm2 and Foxn1 mark early parathyroid- and thymus-specific domains in the developing third pharyngeal pouch. Mech Dev. 103, 141-143.

Gordon J, Xiao S, Hughes B, 3rd, Su DM, Navarre SP, Condie BG, Manley NR (2007). Specific expression of lacZ and cre recombinase in fetal thymic epithelial cells by multiplex gene targeting at the Foxn1 locus. BMC Dev Biol. 7, 69. 
Oh. J. et. al. $\quad$ iTEC-based thymic rejuvenation

Goronzy JJ, Weyand CM (2012). Immune aging and autoimmunity. Cell Mol Life Sci. 69, 1615-1623.

Hale JS, Boursalian TE, Turk GL, Fink PJ (2006). Thymic output in aged mice. Proc Natl Acad Sci U S A. 103, 8447-8452.

Hamazaki Y, Fujita H, Kobayashi T, Choi Y, Scott HS, Matsumoto M, Minato N (2007). Medullary thymic epithelial cells expressing Aire represent a unique lineage derived from cells expressing claudin. Nat Immunol. 8, 304-311.

Hubert FX, Kinkel SA, Davey GM, Phipson B, Mueller SN, Liston A, Proietto AI, Cannon PZ, Forehan S, Smyth GK, Wu L, Goodnow CC, Carbone FR, Scott HS, Heath WR (2011). Aire regulates transfer of antigen from mTEC to dendritic cells for induction of thymic tolerance. Blood.

Itoi M, Tsukamoto N, Amagai T (2007). Expression of Dll4 and CCL25 in Foxn1-negative epithelial cells in the post-natal thymus. Int Immunol. 19, 127-132.

Kim MJ, Miller CM, Shadrach JL, Wagers AJ, Serwold T (2015). Young, proliferative thymic epithelial cells engraft and function in aging thymuses. J Immunol. 194, 4784-4795.

Klein L, Robey EA, Hsieh CS (2019). Central CD4(+) T cell tolerance: deletion versus regulatory T cell differentiation. Nat Rev Immunol. 19, 7-18.

McElhaney JE, Effros RB (2009). Immunosenescence: what does it mean to health outcomes in older adults? Curr Opin Immunol. 21, 418-424.

Min D, Panoskaltsis-Mortari A, Kuro OM, Hollander GA, Blazar BR, Weinberg KI (2007). Sustained thymopoiesis and improvement in functional immunity induced by exogenous KGF administration in murine models of aging. Blood. 109, 2529-2537.

Oh J, Wang W, Thomas R, Su DM (2017). Capacity of tTreg generation is not impaired in the atrophied thymus. PLoS Biol. 15, e2003352.

Ortman CL, Dittmar KA, Witte PL, Le PT (2002). Molecular characterization of the mouse involuted thymus: aberrations in expression of transcription regulators in thymocyte and epithelial compartments. Int Immunol. 14, 813-822.

Palmer S, Albergante L, Blackburn CC, Newman TJ (2018). Thymic involution and rising disease incidence with age. Proc Natl Acad Sci US A.

Prata L, Ovsyannikova IG, Tchkonia T, Kirkland JL (2019). Senescent cell clearance by the immune system: Emerging therapeutic opportunities. Semin Immunol.

Ripen AM, Nitta T, Murata S, Tanaka K, Takahama Y (2011). Ontogeny of thymic cortical epithelial cells expressing the thymoproteasome subunit beta5t. Eur J Immunol. 41, 1278-1287.

Rode I, Martins VC, Kublbeck G, Maltry N, Tessmer C, Rodewald HR (2015). Foxn1 Protein Expression in the Developing, Aging, and Regenerating Thymus. J Immunol. 195, 5678-5687.

Ruan L, Zhang Z, Mu L, Burnley P, Wang L, Coder B, Zhuge Q, Su DM (2014). Biological significance of FoxN1 gain-of-function mutations during T and B lymphopoiesis in juvenile mice. Cell Death Dis. 5, e1457.

Sekai M, Hamazaki Y, Minato N (2014). Medullary thymic epithelial stem cells maintain a functional thymus to ensure lifelong central T cell tolerance. Immunity. 41, 753-761. 
Shimatani K, Nakashima Y, Hattori M, Hamazaki Y, Minato N (2009). PD-1+ memory phenotype CD4+ T cells expressing C/EBPalpha underlie $\mathrm{T}$ cell immunodepression in senescence and leukemia. Proc Natl Acad Sci U S A. 106, 15807-15812.

Sun L, Guo J, Brown R, Amagai T, Zhao Y, Su DM (2010). Declining expression of a single epithelial cellautonomous gene accelerates age-related thymic involution. Aging Cell. 9, 347-357.

Tahir S, Fukushima Y, Sakamoto K, Sato K, Fujita H, Inoue J, Uede T, Hamazaki Y, Hattori M, Minato N (2015). A CD153+CD4+ T follicular cell population with cell-senescence features plays a crucial role in lupus pathogenesis via osteopontin production. J Immunol. 194, 5725-5735.

Thomas R, Wang W, Su DM (2020). Contributions of Age-Related Thymic Involution to Immunosenescence and Inflammaging. Immun Ageing. 17, 2.

Tsai PT, Lee RA, Wu H (2003). BMP4 acts upstream of FGF in modulating thymic stroma and regulating thymopoiesis. Blood. 102, 3947-3953.

Ulyanchenko S, O'Neill KE, Medley T, Farley AM, Vaidya HJ, Cook AM, Blair NF, Blackburn CC (2016). Identification of a Bipotent Epithelial Progenitor Population in the Adult Thymus. Cell reports. 14, 2819-2832.

Vallejo AN (2006). Age-dependent alterations of the T cell repertoire and functional diversity of T cells of the aged. Immunol Res. 36, 221-228.

Wang W, Wang L, Ruan L, Oh J, Dong X, Zhuge Q, Su DM (2018). Extracellular vesicles extracted from young donor serum attenuate inflammaging via partially rejuvenating aged T-cell immunotolerance. FASEB journal : official publication of the Federation of American Societies for Experimental Biology, fj201800059R.

Wertheimer T, Velardi E, Tsai J, Cooper K, Xiao S, Kloss CC, Ottmuller KJ, Mokhtari Z, Brede C, deRoos P, Kinsella S, Palikuqi B, Ginsberg M, Young LF, Kreines F, Lieberman SR, Lazrak A, Guo P, Malard F, Smith OM, Shono Y, Jenq RR, Hanash AM, Nolan DJ, Butler JM, Beilhack A, Manley NR, Rafii S, Dudakov JA, van den Brink MRM (2018). Production of BMP4 by endothelial cells is crucial for endogenous thymic regeneration. Sci Immunol. 3.

Xia J, Wang H, Guo J, Zhang Z, Coder B, Su DM (2012). Age-Related Disruption of Steady-State Thymic Medulla Provokes Autoimmune Phenotype via Perturbing Negative Selection. Aging Dis. 3, 248-259.

Zhang Z, Burnley P, Coder B, Su DM (2012). Insights on FoxN1 biological significance and usages of the "nude" mouse in studies of T-lymphopoiesis. Int J Biol Sci. 8, 1156-1167.

Zook EC, Krishack PA, Zhang S, Zeleznik-Le NJ, Firulli AB, Witte PL, Le PT (2011). Overexpression of Foxn1 attenuates age-associated thymic involution and prevents the expansion of peripheral CD4 memory T cells. Blood. 118, 5723-5731. 
Figure 1. Preparation and characterization of MEFs and iTECs Mouse embryonic fibroblasts (MEFs) were isolated via trypsinized digestion from E13 and E14 embryonic mice, and cultured in plates with or without 4-hydroxy tamoxifen (symbol: xTM). (A). Representative live images from confocal microscopy show MEFs expressed GFP, which represents exogenous FoxN1 (right panels) and was driven by either endogenous FOXN1-carried Cre-recombinase at 3'UTR (FTg:FoxN1Cre; top-right) or R26-carried CreER ${ }^{\mathrm{T}}$ treated with TM (FTg:R26CreER ${ }^{\mathrm{T}} \mathrm{xTM}$; bottom-right); and panels without GFP (left panels) due to either no Cre transgene or no active Cre; (B) Representative flow cytometric dot plots (EpCAM vs. GFP: top panels; and MHC-II vs. GFP: bottom panels), in which MEFs expressing GFP (FTg:R26CreER ${ }^{\mathrm{T}} \mathrm{xTM}$ and $\mathrm{FTg}$ :FoxN1Cre) are termed iTECs (in red boxes of the middle and right panels), compared to MEFs that did not express GFP (FTg:R26CreER ${ }^{\mathrm{T}}$ due to Cre inactivated without TM treatment - left panels); (C) Summarized gene (FoxN1, Dll4, and Ccl25) expression (via RT-PCR) in cells of four groups: (1) FTg-only: without Cre; (2) FTg:FoxN1Cre: Cre expression was endogenously turned on in FoxN1 ${ }^{+}$cells; (3) FTg:R26CreER ${ }^{\mathrm{T}}$ xTM: Cre was activated via TM induction, and (4) a newborn thymus control group. A Student $t$-test was used to determine statistical significance and $P$ values are shown between every two groups. In addition, an ordinary one-way ANOVA $p$-value summary by comparing multiple groups is shown on top of each panel. All $p$-values were calculated by mean \pm SD and " $n$ " animal numbers. Scales showed in each bar are SEMs. Each symbol represents cells from an individual embryonic sample.

Figure 2. Transplantation of iTECs drove re-growth of the aged thymus in both male and female mice Naturally aged mice (WT, $\geq 18$ months old at cellular transplantation; $20-21$ months old at analysis) were intra-/peri-thymically (i.t./p.t.) transplanted with FTg-only MEFs or either of two promoter-driven exogenous FoxN1 expressing iTECs; one group of young mice served as a control. Forty-five days after engraftment, the thymic mass was analyzed. (A) Representative images of the thymuses engrafted with donor cells; (B) Ratios of thymus/body weight and (C) Results of absolute thymocyte numbers per thymus from donor cellengrafted aged male and female mice (one young group, leftmost, served as control); Statistical analysis, data expression, and each symbol per animal are the same as Fig. 1.

Figure 3. Transplantation of iTECs rejuvenated thymic architecture of aged mice via both exogenous iTEC growth and endogenous TEC regrowth Same experimental setting as described in Fig. 2. Cryosections of the thymic tissue (Representative immunofluorescence images shown in panels A-D) were co-stained with various immunofluorescence antibodies for TEC developmental and architectural profiles. (A) K5 (red) vs. K8 (green); (B) UEA-1 (red) vs. K8 (green); (C) Claudin (Cld)-3+4 (red) vs. K8 (green); (D) $\beta 5 \mathrm{t}$ (red) vs. K8 (green). Data are representative of 3 biological replicates in each group with essentially identical results. (E) Flow cytometric analysis of endogenous TECs (FoxN1 $1^{+} \mathrm{GFP}^{- \text {neg }}$ ) and exogenous TECs (from iTECs, FoxN1 $1^{+} \mathrm{GFP}^{+}$) in the mTECs $\left(\mathrm{CD} 45^{-} \mathrm{MHC}-\mathrm{II}^{+}\right)$or pan-TECs $\left(\mathrm{CD} 45^{-} \mathrm{EpCam}^{+}\right)$of various thymuses, 10 or 20 days after engraftment with MEFs or iTECs, based on endogenous FoxN1 (by antibody) and exogenous FoxN1 (by GFP) expression.

Figure 4. Transplantation of iTECs boosted Aire gene expression in the age thymus and showed enhanced negative selection signaling strength via Nur77 in $\mathrm{CD}^{\mathrm{SP}}$ thymocytes of aged mice Same experimental setting as described in Fig. 2. (A) Representative immunofluorescence staining images of Aire ${ }^{+}$ TECs (red) in $\mathrm{K}^{+}$TEC counterstaining (green). Data are representative of three biological replicates in each group with essentially identical results; (B) Summarized result shows the percent area of Aire ${ }^{+}$TECs against $\mathrm{K} 8^{+}$counterstaining based on the slides in panel A. Each symbol represents one thymic tissue section; \pm 6 thymic tissue sections at disconnected locations (non-sequential slides) from an individual mouse thymus were counted with Image-J software; (C) Flow cytometric results show increased Nur77 signaling strength [relative quantitative (RQ) mean fluorescent intensity (MFI)] in $\mathrm{CD}^{\mathrm{SP}}$ thymocytes of young (control) or 
Oh. J. et. al. $\quad$ iTEC-based thymic rejuvenation

599 aged mice that were engrafted with MEFs or two types of iTECs. Left panel: histogram of Nur77 MFI in 600 CD4 ${ }^{\text {SP }}$ thymocytes; Right panel: Nur77 RQ-MFI in CD4 ${ }^{\text {SP }}$ populations of various groups. Statistical analysis, 601 data expression, and each symbol per animal are the same as Fig. 1.

602

603

604

605

606

607

608

609

610

611

612

613

614

615

616

617

618

619

620

621

622

623

624

625

626

627
Figure 5. Transplantation of iTECs partially rescued declined thymocyte negative selection in aged mice (A) Reconstituted mOVA-Tg aged mice (mOVA-Tg young mice for control) with OT-II TCR-Tg bone marrow (BM) cells via a $2900 \mathrm{Rad}$ irradiation were intra-/peri-thymically transplanted with MEFs (FTg-only) or iTECs (FTg:FoxN1Cre). Negative selection of OT-II TCR-Tg specific CD4 ${ }^{\mathrm{SP}}\left(\mathrm{CD} 4^{+} \mathrm{CD}{ }^{\text {-neg }}\right)$ thymocytes in the host mOVA-Tg TEC microenvironment was analyzed with a flow cytometer. (B) Flow cytometric gating scheme of CD4 vs CD8 (top row) and engrafted donor BM (CD45.1 $1^{+}$) produced OT-II specific TCR$\mathrm{Tg} \mathrm{CD}^{\mathrm{SP}}$ thymocytes (bottom row). (C) Summarized results of \% OT-II specific TCR-Tg CD4 ${ }^{\mathrm{SP}}$ thymocytes. (D) A representative histogram of Nur77 MFI in OT-II specific TCR-Tg CD4 ${ }^{\text {SP }}$ thymocytes. (E) Relative quantitative (RQ)-mean fluorescent intensity (MFI) of Nur77 signaling strength in OT-II specific TCR-Tg CD4 ${ }^{\text {SP }}$ thymocytes, by setting RQ-MFI in young thymocytes as 1.0 (i.e. signaling with $100 \%$ intensity). Statistical analysis, data expression, and each symbol per animal are the same as Fig. 1.

\section{Figure 6. Transplantation of iTECs attenuated inflammaging-associated phenotypes by reducing} inflammatory cytokines and lymphoid cell infiltration into non-lymphoid organs in aged mice (A) Serum was collected from mice with the same treatment as in Fig. 2. Concentration of pro-inflammatory cytokines IL-6 (Left panel) and IL-1 $\beta$ (Right panel) in pg/mg of serum protein was measured through an ELISA method. Statistical analysis, data expression, and each symbol per animal are the same as Fig. 1. (B) Workflow of adoptive transfer, showing that splenocytes $\left(2.5 \times 10^{7}\right.$ cells per recipient mouse $)$ from rejuvenated and control young or aged WT mice were transferred via i.v. injection into young $\operatorname{Rag}^{-/-}$recipient $^{-}$ mice. Eight weeks after the transfer, the salivary glands were subjected to analysis of lymphocyte infiltration; (C) Representative H\&E stained images of the salivary glands from the adoptive transfer Rag $^{-/-}$recipient mice, showing foci of lymphoid cell infiltration (red circles in 4x images and yellow circles in 20x images). Data are representative of 500 tissue slides from 3 animals in each group, and numbers of infiltration foci in 500 tissue slides and the \% of lymphoid cell infiltrated foci are shown. 
bioRxiv preprint doi: https://doi.org/10.1101/2020.03.17.995357; this version posted March 18, 2020. The copyright holder for this preprint (which was not certified by peer review) is the author/funder. All rights reserved. No reuse allowed without permission.

A.
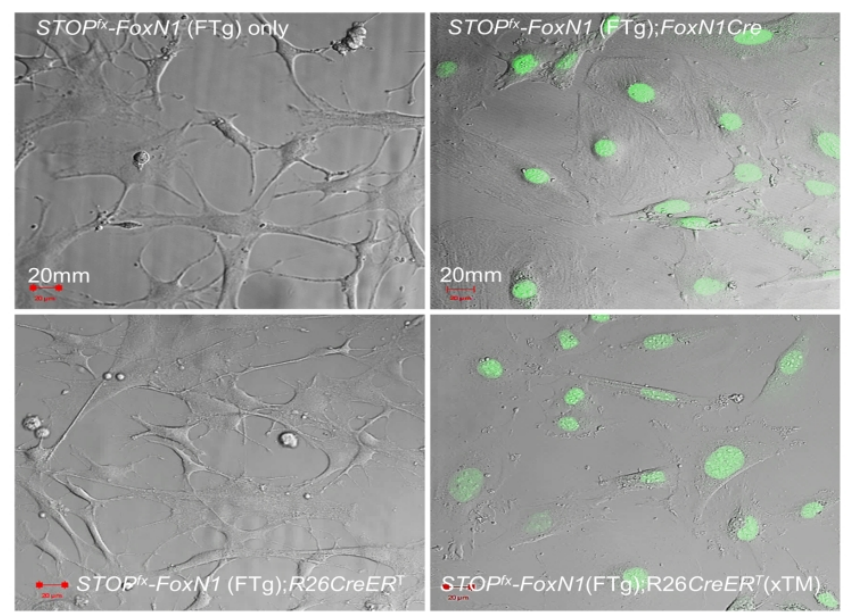

B.

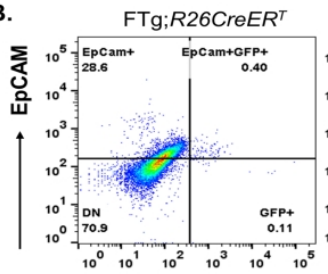

FTg;R26CreER ${ }^{T}(\mathrm{xTM})$

FTg;FoxN1Cre
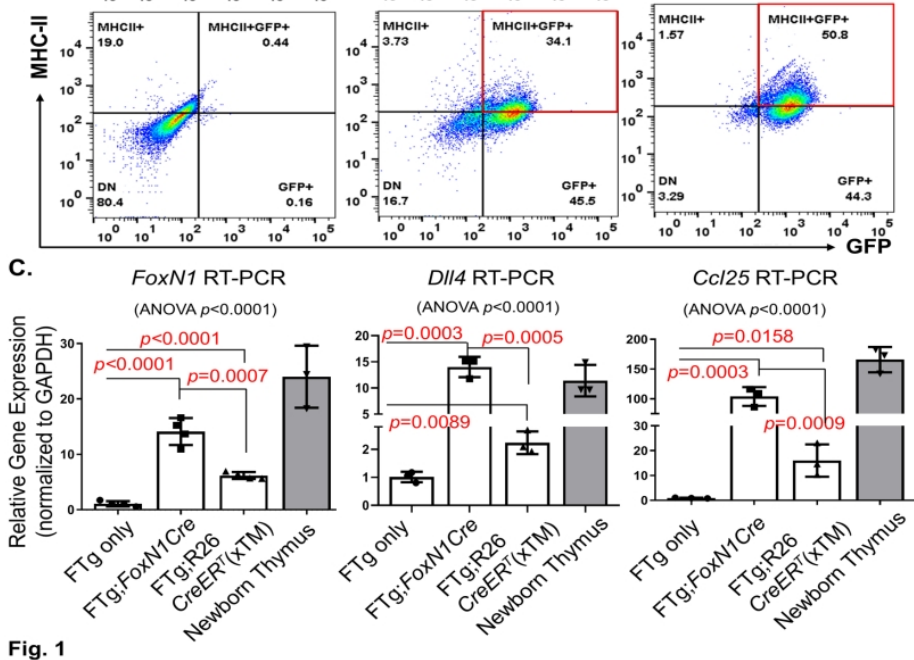

Fig. 1 


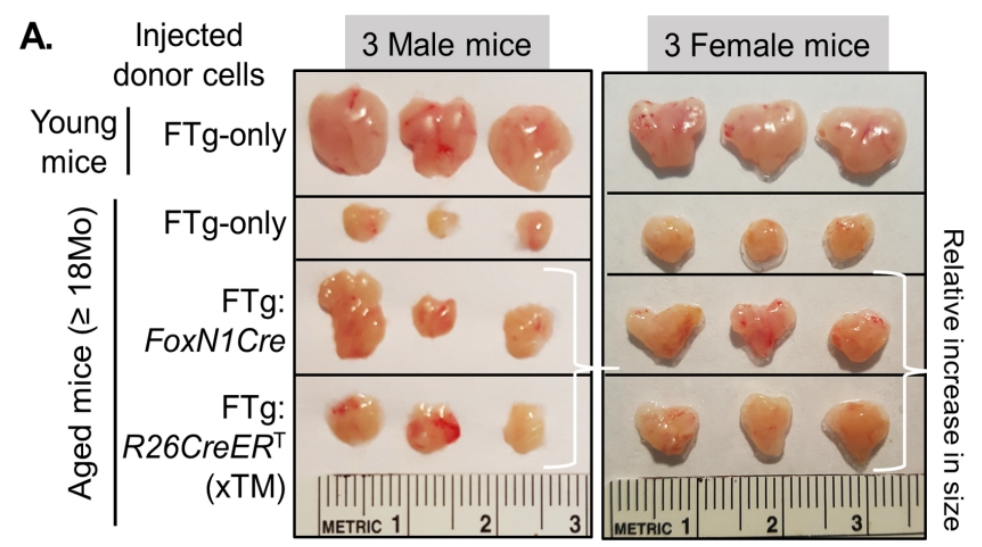

B.
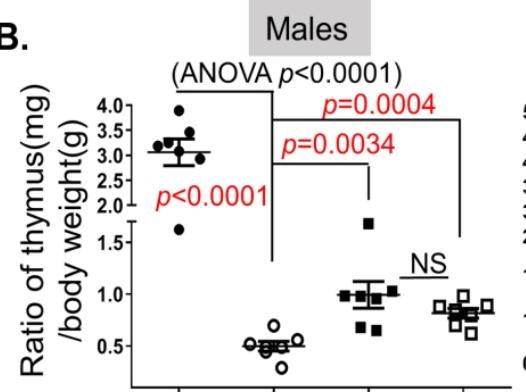

c.
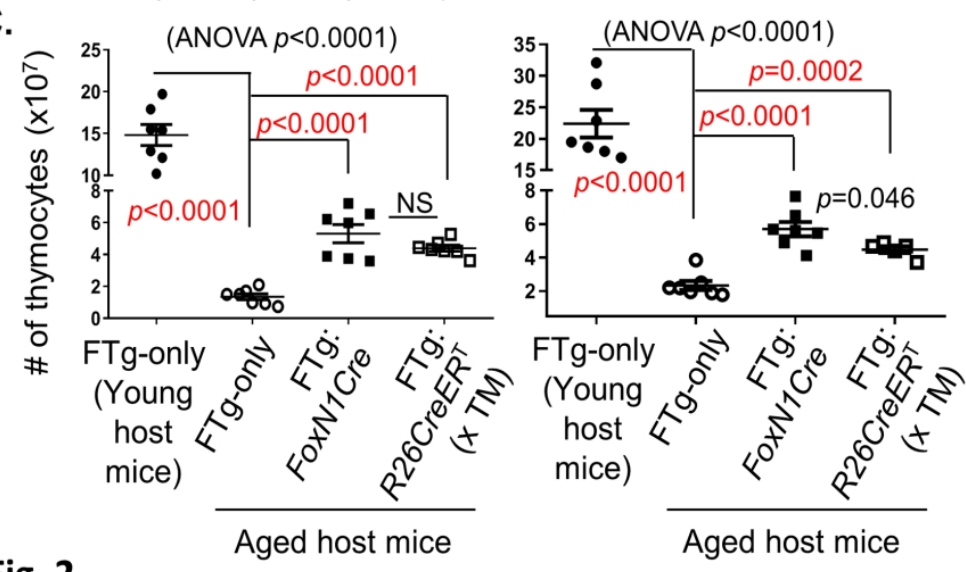

Fig. 2. 
bioRxiv preprint doi: https://doi.org/10.1101/2020.03.17.995357; this version posted March 18, 2020. The copyright holder for this preprint (which was not certified by peer review) is the author/funder. All rights reserved. No reuse allowed without permission.

Thymus from young mice

Thymuses from aged $(\geq 18 \mathrm{Mo})$ mice

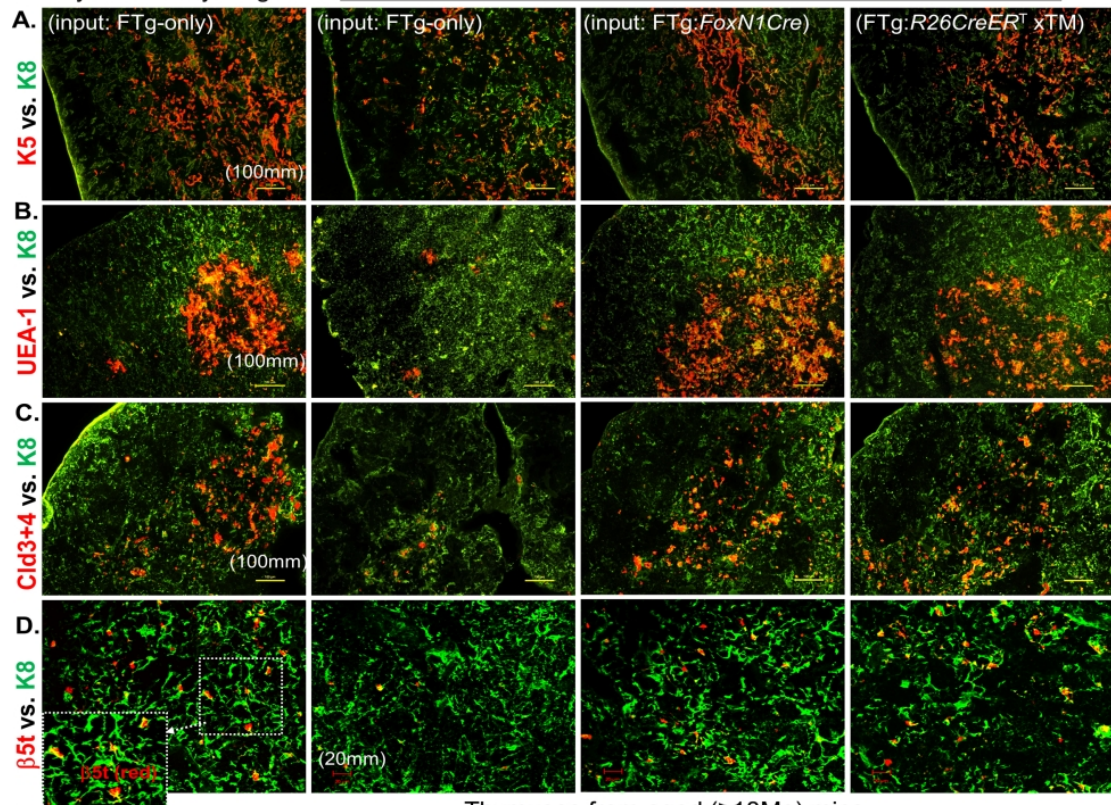

E.

Thymuses from aged $(\geq 18 \mathrm{Mo}$ ) mice

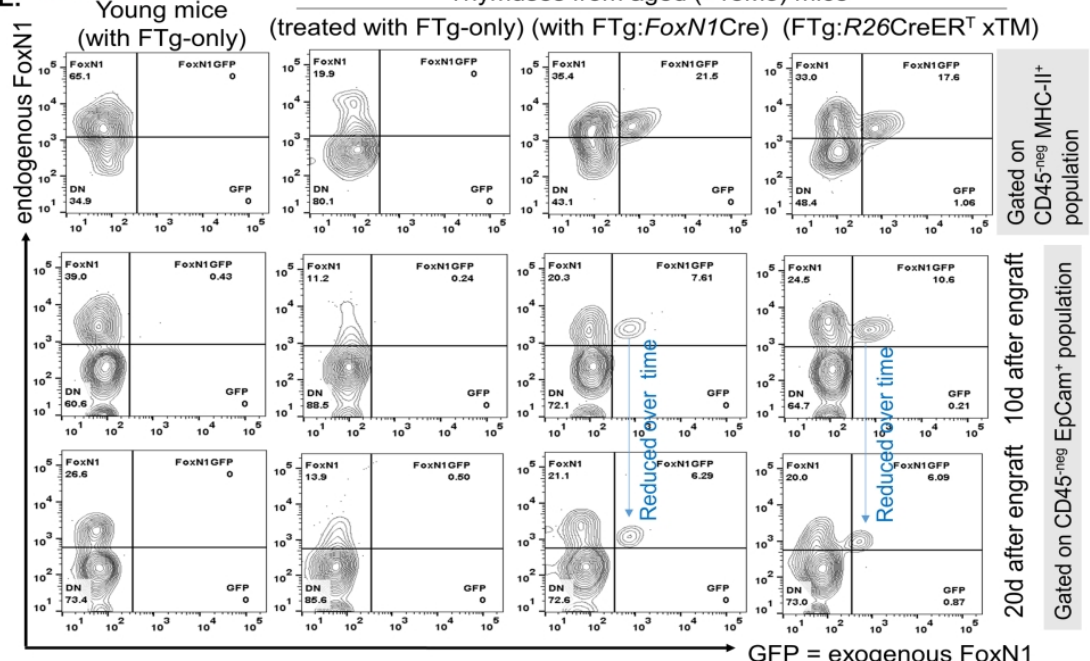

Fig. 3. 


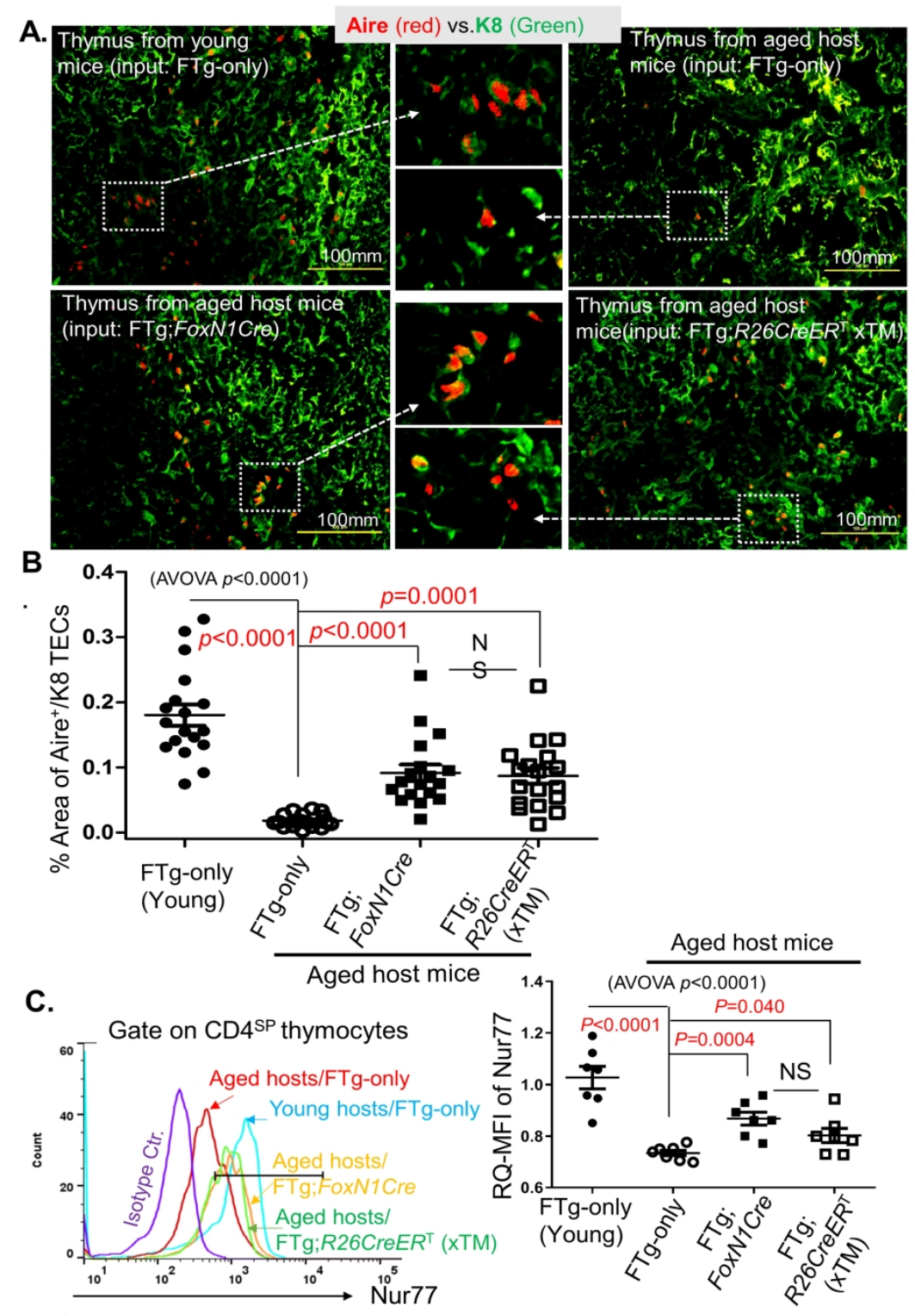

Fig. 4. 
A.

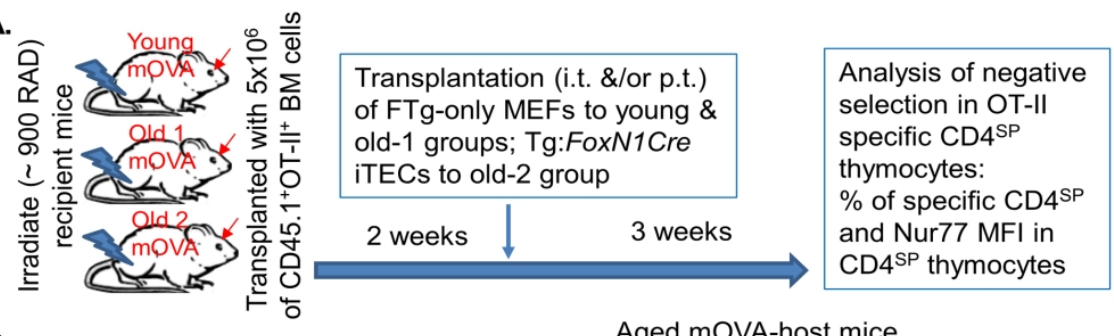

B.

Young mOVA-host mice

Aged mOVA-host mice

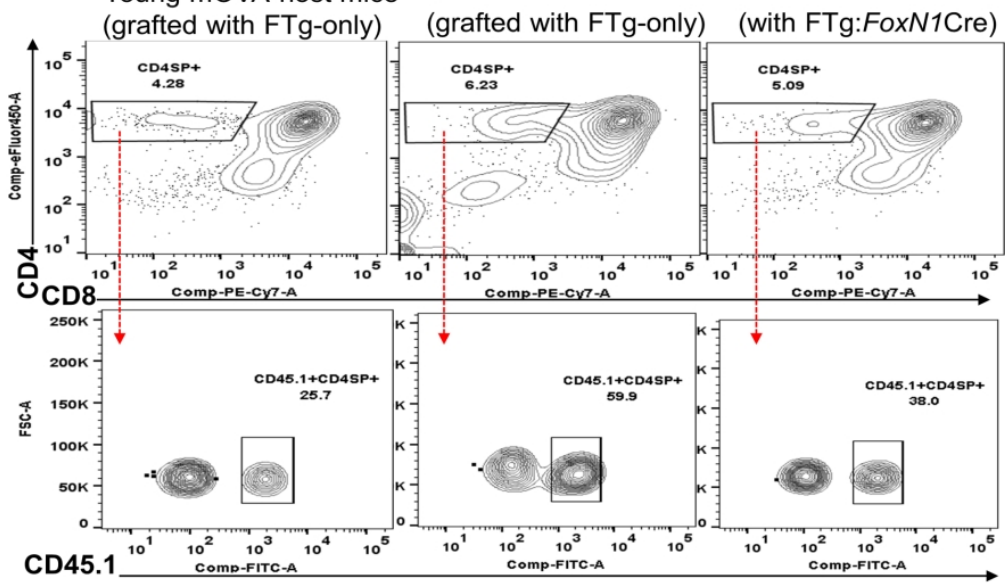

C. (AVOVA $p<0.010)$

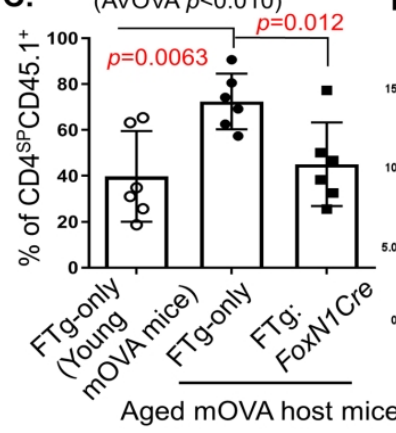

D. Gate on $\mathrm{CD}^{\mathrm{SPCD}}{ }^{\mathrm{C}} \mathrm{5.1}$ + $^{+}$

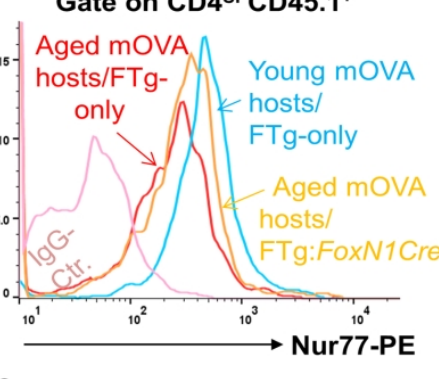

Fig. 5

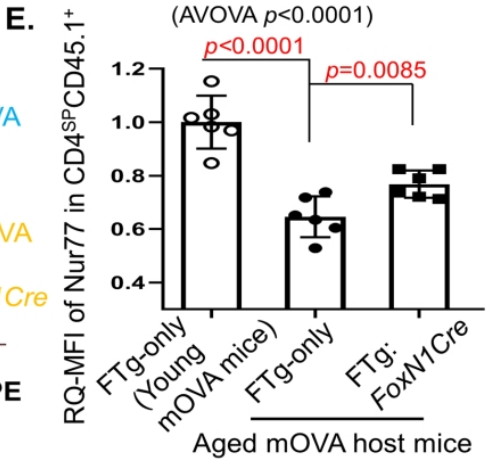


bioRxiv preprint doi: https://doi.org/10.1101/2020.03.17.995357; this version posted March 18, 2020. The copyright holder for this preprint (which was not certified by peer review) is the author/funder. All rights reserved. No reuse allowed without permission.

A.
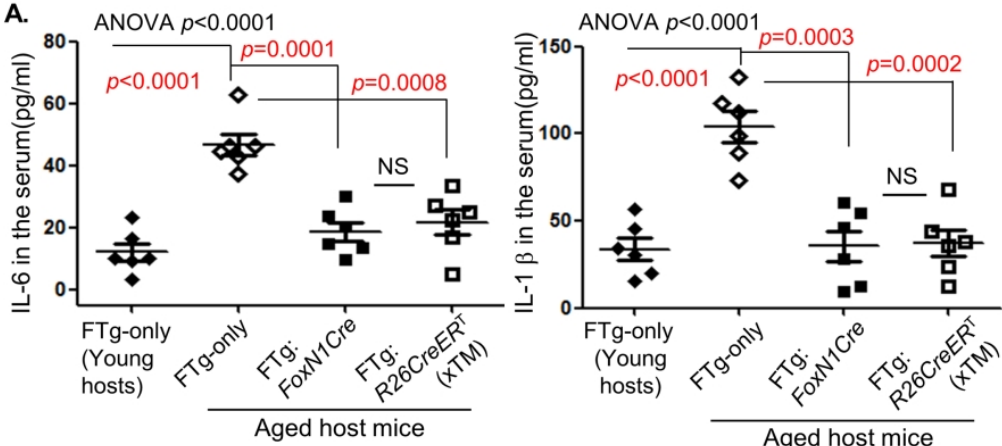

B.

C.

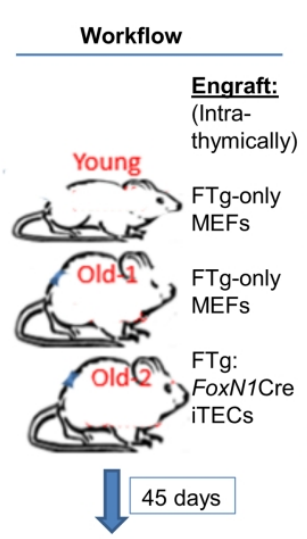

Isolated splenocytes; Adoptive transfer:

(intravenously) into

$\mathrm{Rag}^{\prime-}$ young host mice

(2.5 $\times 10^{7} /$ recipient)

8 weeks

Dissected the salivary gland from $\mathrm{Rag}^{/-}$mice;

H \& E staining:

Observed and analyzed

lymphoid infiltration

(recorded focus numbers

in 500 tissue slides)

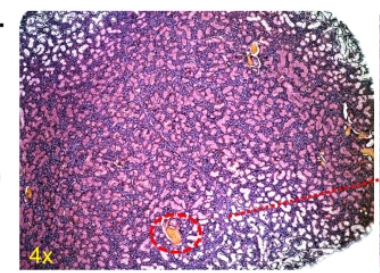

Adoptive transfer: Splenocytes

from young hosts/FTg-only MEFs (foci $=7 / 500=1.4 \%$ )
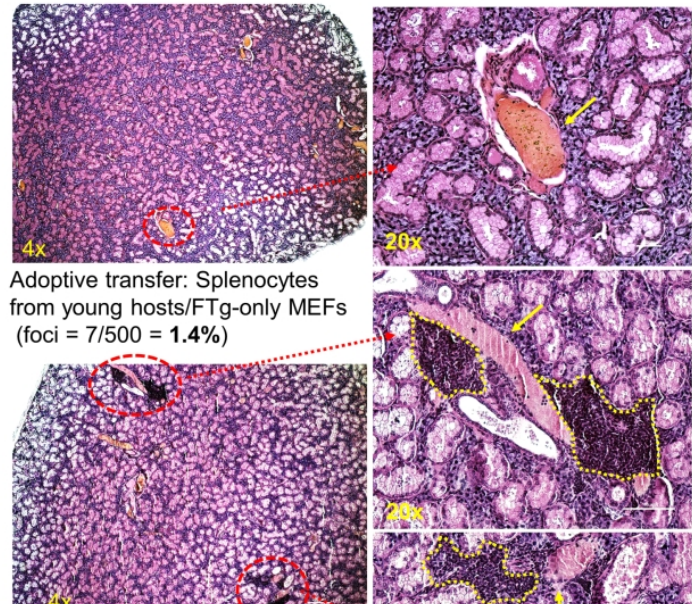

Adoptive transfer: Splenocytes from old-1 hosts/FTg-only MEFs (foci $=218 / 500=43.6 \%$ )

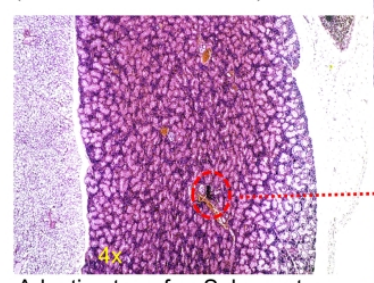

Adoptive transfer: Splenocytes from old-2 hosts/FTg:FoxN1Cre iTECs $($ foci $=126 / 500=\mathbf{2 5 . 2} \%$ )
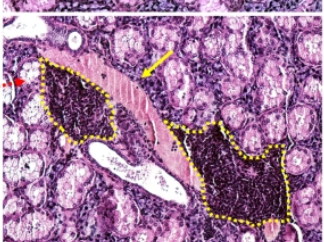

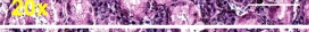
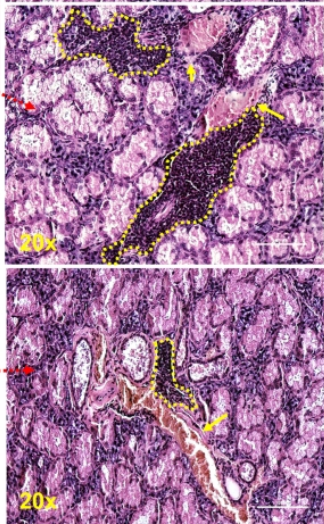

Fig. 6 